\title{
Machine learning methods for crop chlorophyll variable retrieval
}

\author{
Fenghua $\mathrm{Yu}^{1,2}$, Tongyu $\mathrm{Xu}^{1,2^{*}}$, Chunling Chen ${ }^{1,2}$, Wen $\mathrm{Du}^{1}$, Shuai Feng ${ }^{1}$ \\ (1. College of Information and Electrical Engineering, Shenyang Agricultural University, Shenyang 110866, China; \\ 2. Liaoning Engineering Research Center for Information Technology in Agriculture, Shenyang 110866, China)
}

\begin{abstract}
Hyperspectral remote sensing technology improves the retrieval ability of chlorophyll content in crops. The machine learning method has been developed and applied to crop phenotyping information inversion. This study combined radiative transfer model (PROSPECT-4) and Gauss Process Regression algorithm (GPR) to retrieval crop leaf chlorophyll content. The test was conducted in the eastern city of Shenyang, Liaoning Province, China with a japonica rice. This paper describes (1) The PROSPECT-4 model was analyzed by GSA tool, and the sensitivity band range of crop chlorophyll was at $400-750 \mathrm{~nm}$. (2) The chlorophyll content model was established with great accuracy $\left(R^{2}=0.8638\right)$ that can predict the crop leaf chlorophyll content; (3) The results demonstrated that crop chlorophyll is inversion by PROSPECT model and machine learning algorithm. Therefore, crop chlorophyll content can be estimated by hyperspectral data that may be used for crop growth management. This research can provide an efficient method to detect crop leaf chlorophyll content at the RTMS in the future. Keywords: Gaussian Processes Regression, hyperspectral remote sensing, leaf chlorophyll, machine learning, PROSPECT
\end{abstract} DOI: $10.33440 /$ j.ijpaa.20180101.0006

Citation: Yu F H, Xu T Y, Chen C L, Du W, Feng S A. Machine learning methods for crop chlorophyll variable retrieval. Int J Precis Agric Aviat, 2018; 1(1): 32-36.

\section{Introduction}

Hyperspectral remote sensing is characterized by many bands and narrow band, which is a crucial technology for the development of precision agriculture ${ }^{[1]}$. Precision agriculture requires fine management of farmland, so remote sensing technology, including hyperspectral, thermal imaging and LiDAR systems, which provides technical support for the implementation of precision agriculture. Methods using hyperspectral remote sensing technology are particularly promising as they allow for non-invasive, fast and automated measurements with both spatial and temporal resolution in the field. They are based on transmittance, reflectance signals from the plants, which contain information about agronomic and physiological traits ${ }^{[2]}$. Having access to operationally acquired imaging spectroscopy data with hundreds of bands paves the path for a wide variety of monitoring applications, such as the biochemical vegetation properties ${ }^{[3]}$. In the process of crop growth, its canopy structure, physiological characteristics, environmental background and so on will change, resulting in changes in leaf and canopy spectrum. It is based on crop differences in spectral response to monitor their growth.

Such large number of data dimensions induces an important methodological challenge. Hyperspectral remote image data include highly correlated and noisy spectral bands, and frequently

\section{Received date: 2018-05-18 Accepted date: 2018-08-18}

Biographies: Fenghua Yu, PhD, Lecturer, research interests: remote sensing application in agriculture, Email: adan@syau.edu.cn; Chunling Chen, PhD, Professor, research interests: agricultural remote sensing, Email: 342082281@qq.com; Wen Du, PhD, research interests: agricultural remote sensing, Email: duwen@ syau.edu.cn; Shuai Feng, PhD, Canditate, research interests: artificial intelligence, Email: 185888819@qq.com.

*Corresponding author: Tongyu $\mathrm{Xu}, \mathrm{PhD}$, Professor, Dean, research interests: Intelligent agriculture. College of Information and Electrical Engineering, Shenyang Agricultural University, Shenyang 110866, China. Email: yatongmu@163.com. create statistical problems (e.g., the Hughes effect) due to small sample sizes compared to the large number of available, possibly redundant, spectral bands ${ }^{[4]}$. These characteristics may lead to a violation of basic assumptions behind statistical models or may affect the model outcome. Models fitted with such multi-collinear data sets are prone to over-fitting, and transfer to other scenarios may thus be limited. Naturally, these issues affect the prediction accuracy as well as the interpretability of the regression (retrieval) models ${ }^{[5]}$. Therefore, how to reduce the hyperspectral dimension and optimize the spectral, select the most suitable inversion of crop physiological information parameters, is the priorities of using the hyperspectral to carry out research.

Chlorophyll is a class of photosynthesis related to the most important pigment. Photosynthesis is the process of converting light energy into chemical energy by synthesizing some organic compounds $^{[6]}$. Chlorophyll absorbs energy from light, and $t$ convert it into carbon dioxide to carbohydrates. It is a key factor in regulating the biophysical and physiological processes of crops $^{[7]}$. Estimating NDVI model of rice leaves based on NDVI and environmental data of rice canopy. The results show that NDVI of rice leaves is highly correlated with canopy NDVI and multi-source environmental data ${ }^{[8]}$. Traditional methods of measuring chlorophyll in the laboratory, includes collecting crop leaf samples for chemical analysis, not only require destruction of the crop leaf samples but are also labor-intensive and expensive ${ }^{[9]}$. Currently, remote sensing techniques have been proposed for monitoring the crop chlorophyll. In recent years, the main method has been developed for remote estimation of chlorophyll status is empirical relationships between chlorophyll and vegetation indices (VIs). Crop leaf has a strong absorption characteristic in the visible light red band, strongly reflects near infrared band, which is the vegetation remote sensing monitoring using satellite band detection data from a combination of can reflect the crop growth index ${ }^{[10]}$. Different vegetation combinations can be obtained by different combinations of these 
two bands vegetation index. Vegetation index was used to invert chlorophyll content mainly through statistical methods. Chappelle used a narrow band vegetation index to determine the content of chlorophyll of leaves ${ }^{[11,12]}$. Blackburn found a number of spectral indices for estimating pigment concentrations at the leaf scale. The results indicate that the optimal wavebands for chlorophyll estimation are identified empirically at $680 \mathrm{~nm}$ and $635 \mathrm{~nm}$. Two new indices (PSSR and PSND) that were developed had the strongest and most linear relations with chlorophyll concentrations ${ }^{[13]}$. However, there are still some problems in the retrieval of chlorophyll content using vegetation index, Including NDVI, PRI and Green Normalized Difference Vegetation Index (GNDVI) or other vegetation index are likely to be saturated during the inversion process of chlorophyll, resulting in a decrease of inversion accuracy. The model established by using the vegetation index to invert the chlorophyll content of the crop is limited by the experimental conditions, the testing instrument and other factors.

Another method of inverting chlorophyll is radiative transfer models (RTMs) ${ }^{[14,15]}$. Given spectra, find the closest spectra in the database and return the corresponding parameter. Two main approaches: (i) Minimizes a function that calculates the RMSE between the measured and estimated quantities by successive input parameter iteration. (ii) Precompute the model reflectance for a large range of combinations of parameter values, so the problem reduces to searching a LUT for the modeled reflectance that most resembles the measured one ${ }^{[16,17]}$.

The objectives of this work are therefore threefold: (1) The global sensitivity analysis of the prospect model was carried out using the global sensitivity analysis tool to find the hyperspectral spectral range affected by chlorophyll; (2) To compare the accuracy of the chlorophyll inversion model established by different machine learning methods; (3) to establish hyperspectral inversion models for chlorophyll.

\section{Materials and methods}

\subsection{Experimental data}

We choose the LOPEX 93 as the experimental database for this study. LOPEX 93 database including many crops biochemical information and leaf hyperspectral reflectance. The band range from $400 \mathrm{~nm}$ to $2500 \mathrm{~nm}$. About 70 leaf samples representative of more than 50 species of woody and herbaceous plants in this database ${ }^{[18]}$.

The crop parameters measured by the LOPEX 93 database include $\mathrm{N}$, chlorophyll, water, biomass, and other input parameters required by the PROSPECT model. This database also including crop leaf high resolution visible and near infrared reflectance spectroscopy.

\subsection{Crop leaf radiative transfer model}

In this research we choice PROSPECT model for inversion of crop chlorophyll. PROSPECT is a radiative transfer model based on Allen`s generalized "plate model" that represents the optical properties of plant leaves from $400 \mathrm{~nm}$ to $2500 \mathrm{~nm}$ Scattering is described by a spectral refractive index (n) and a parameter characterizing the leaf mesophyll structure $(\mathrm{N})$. Absorption is modeled using pigment concentration $\left(\mathrm{C}_{\mathrm{a}+\mathrm{b}}\right)$, water content $\left(\mathrm{C}_{\mathrm{w}}\right)$, and the corresponding specific spectral absorption coefficients $\left(K_{a+b}\right.$ and $\left.K_{w}\right)$. The parameters $n, K_{a+b}$, and $K_{w}$ have been fitted (using experimental data corresponding to a wide range of plant types and status. PROSPECT has been tested successfully on independent data sets. Its inversion allows one to reconstruct, with reasonable accuracy, leaf reflectance, and transmittance features in the $400-2500 \mathrm{~nm}$ range by adjusting the three input variables $\mathrm{N}, \mathrm{C}_{\mathrm{a}+\mathrm{b}}$, and $\mathrm{C}_{\mathrm{w}}{ }^{[19]}$.

The principle of the model is as follows: The interaction of electromagnetic radiation with plant leaves (reflection, transmission, absorption) is dependent on the chemical and physical properties of the leaves ${ }^{[20]}$. In the visible light band, the light absorption is essentially formed by the rotation and movement of electrons in chlorophyll $\mathrm{a}$, chlorophyll $\mathrm{b}$ and other pigments; in the near-infrared and mid-infrared bands, it is mainly formed by the vibration and rotation of electrons in water ${ }^{[21]}$. Refractive index $\mathrm{n}$ is not continuous in the blade, $n=1.4$ for water-containing cell walls, $n=1.33$ for water and $n=1$ for air, so the internal biochemical composition and structural properties of the whole spectrum the leaf reflectivity and transmittance of the band $^{[22]}$.

\subsection{Hyperspectral remote sensing data Global Sensitivity Analysis(GSA)}

An important requirement is to know the key input variables driving the spectral output in a specific spectral region. Such knowledge can lead to a simplified model that is driven only by the key variables, which makes exploration of a broad range of target and observation conditions easier and more effective ${ }^{[23]}$. To achieve this, GSA is required. The global sensitivity analysis is performed over the entire parameter range, and the effect of coupling between different parameters on the output of the model is considered, which is very suitable for the sensitivity analysis of complex nonlinear models ${ }^{[24]}$.

The most popular global sensitivity analysis method is Sobol algorithm $^{[25]}$. This work the variance-based sensitivity measures was used. The algorithm are represented as follows:

$$
\begin{gathered}
1=\sum_{i} S_{i}+\sum_{i} S_{i} \sum_{j>i} S_{i j}+, \ldots,+S_{12, \ldots, k} \\
S_{i}=\frac{V_{i}}{V(Y)}=\frac{V\left[E\left(Y \mid X_{i}\right)\right]}{V(Y)}
\end{gathered}
$$

In this equation, $S_{i}, S_{i j}, \ldots, S_{12, \ldots, k}$ are Sobol's global sensitivity indices. Where $S_{i}$ and represent the first order sensitivity index, whereas, $S_{i j}, \ldots, S_{12, \ldots, k}$ are the sensitivity measures for the higher order terms. Output $Y$ to the input parameter $X_{i}$ (without interaction terms). The total effect sensitivity index $S_{T i}$, measures the whole effect of the variable:

$$
S_{T i}=S_{i}+\sum_{J \neq i} S_{i j}+\cdots=\frac{E\left[V\left(Y \mid X_{\sim i}\right)\right]}{V(Y)}
$$

GSA useful to identify RTM key and non-influential variables $^{[26]}$.

\subsection{Gaussian processes regression}

Traditional data modeling methods commonly used in the process are Estimation, regression and function approximation. With the continuous improvement of computer computing ability, the current research on machine learning has become a trend. Such as the Gaussian Processes regression $(\mathrm{GPR})^{[27,28]}$, in which we will focus here.

GPR can be transformed into a linear relation by mapping the data of non-linear relation to the characteristic space by the way of nucleus substitution, so that the complex nonlinear problem can be transformed into a linear problem. A key step in the use of GPR modeling is to determine the kernel function. Gaussian processes 
can choose different types of kernel functions. Each kernel function has different structures, and its ability to describe data is different. The nature of GPR model is also determined by its kernel function ${ }^{[29]}$. The kernel functions of the GPR model include the mean kernel function and the covariance kernel function. The mean function $m(x ; \Phi)$ can be used to denote the mathematical expectation of the function $y(x)$ for which $x$ is input without observations. In general, Take $m(x ; \Phi)=0$, the zero mean function, which means that the initial output of the function under any input data is ideally zero. It is also possible to assume that the mean function is a constant that is not zero, and that this constant constitutes a super parameter with respect to the prior probability. The covariance function $K\left(x_{1}, x_{2} ; \Phi\right)$ is the center of the stochastic output variable corresponding to two stochastic input points in space. It is a key factor to measure the similarity or correlation degree between different samples, which is the key factor influencing the prediction effect of GPR model. Commonly used covariance kernel functions include Squared exponential covariance function (SE), Matem covariance function, Rational square covariance function (RQ), Periodic covariance function (PER). We use the SE kernel function in this work:

$$
k\left(x_{i}, x_{j}\right)=\sqrt{\pi} l \sigma_{p}^{2} \exp \left(-\frac{\left(x_{i}-x_{j}\right)^{2}}{2(\sqrt{2} l)^{2}}\right)
$$

where, $\sigma$ is output size parameters; $l$ is length scale parameter; $x_{i}$ and $x_{j}$ are the input spectrum. If $x_{i} \approx x_{j}$, then $k\left(x_{i}, x_{j}\right)$ takes the maximum, which indicates that the two functions are close. If the difference between $x_{i}$ and $x_{j}$ gradually become larger, $k\left(x_{i}, x_{j}\right)$ approaching zero, which means that two points farther and farther apart, at this time $\sqrt{2} l$ determines its distance from far and near effect. Hence, low values of $\sqrt{2} l$ indicate a higher informative content of this certain per input bands to the training function $k$.

This $\sqrt{2} l$ property shall be further exploited in this paper.

\subsection{ARTMO tools}

The automated radiative transfer models operator (ARTMO) graphic user interface (GUI) is a software package that provides essential tools for running and inverting a suite of plant RTMs, both at the leaf and at the canopy level. ARTMO facilitates consistent and intuitive user interaction, thereby streamlining model setup, running, storing and spectra output plotting for any kind of optical sensor operating in the visible, near-infrared and shortwave infrared range $(400-2500 \mathrm{~nm})^{[30]}$.

\section{Results and discussion}

\subsection{GSA reflectance results}

Sobol's First order sensitivity index $\left(\mathrm{S}_{\mathrm{Fi}}\right)$ and Total order sensitivity index $\left(\mathrm{S}_{\mathrm{Ti}}\right)$ results on surface reflectance across the 400-2400 nm region are given in Figure 1. The PROSPECT-4 model has four input variables, include leaf structural parameter $(\mathrm{N})$, leaf chlorophyll content (Cab), leaf equivalent water thickness (EWT) and leaf dry matter content (DW). The GSA results revealed that four input variables drive the PROSPECT-4 reflectance.

The leaf chlorophyll $\mathrm{a}+\mathrm{b}$ content $(\mathrm{Cab})$ only governed over $70 \%$ of variation in reflectance at wavelengths in the range of $400 \mathrm{~nm}$ to $750 \mathrm{~nm}$. In this band range, the leaf structural parameter $(\mathrm{N})$ governed less than $20 \%$ of variation in reflectance, and leaf dry matter content (DW) governed less than $10 \%$ of variation in reflectance. As shown in Figure 1, moisture has no sensitive band in 400-750 nm. Apart from EWT, spectral features in the visible part were controlled primarily by Cab, $\mathrm{N}$ and DW. The 400-750 $\mathrm{nm}$ spectral window is the photosynthetically active radiation for plants with $\mathrm{Cab}$ as the main absorbing pigment.

Figure 2 shows the dominant range of chlorophyll band, from the figure can be seen, to use spectral information inversion of crop chlorophyll content, only need to use $400-750 \mathrm{~nm}$ to carry out research, do not need to $400-2500 \mathrm{~nm}$ all Bands are used for modeling, reducing the dimensionality of the data.

\subsection{GPR results}

Figure 3 shows the comparison of predicted and measured values of chlorophyll inversion using GPR. Regarding chlorophyll retrieval with GPR, the $R^{2}=0.8638, R M S E=0.9435$. It can be seen from the values of $\mathrm{R}^{2}$ and RMSE that it is ideal to use the prospect model to retrieve the chlorophyll content using the method of GPR.

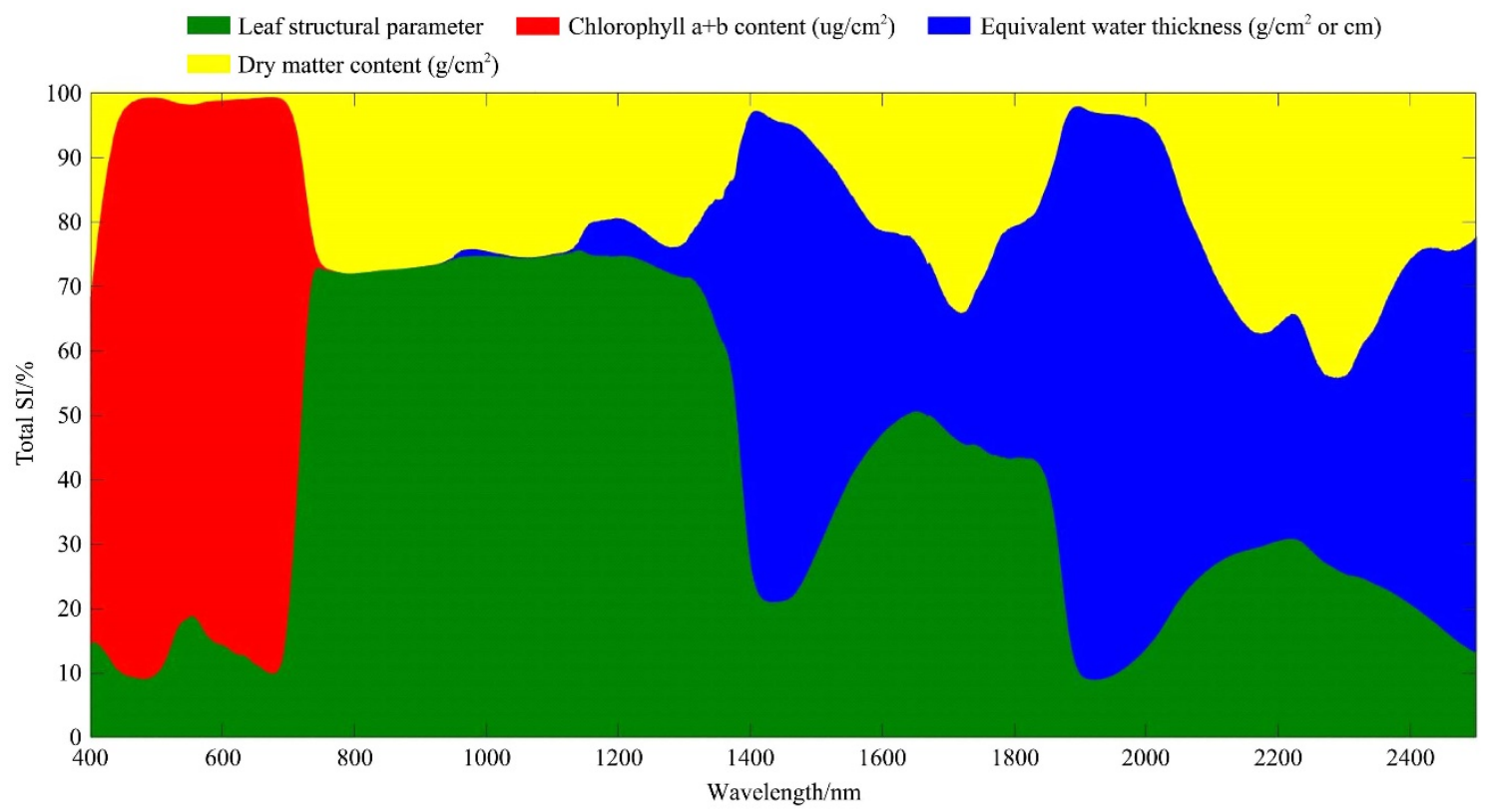

Figure 1 GSA Total sensitivity index $\left(\mathrm{S}_{\mathrm{Ti}}\right)$ for PROSPECT4 model reflectance 


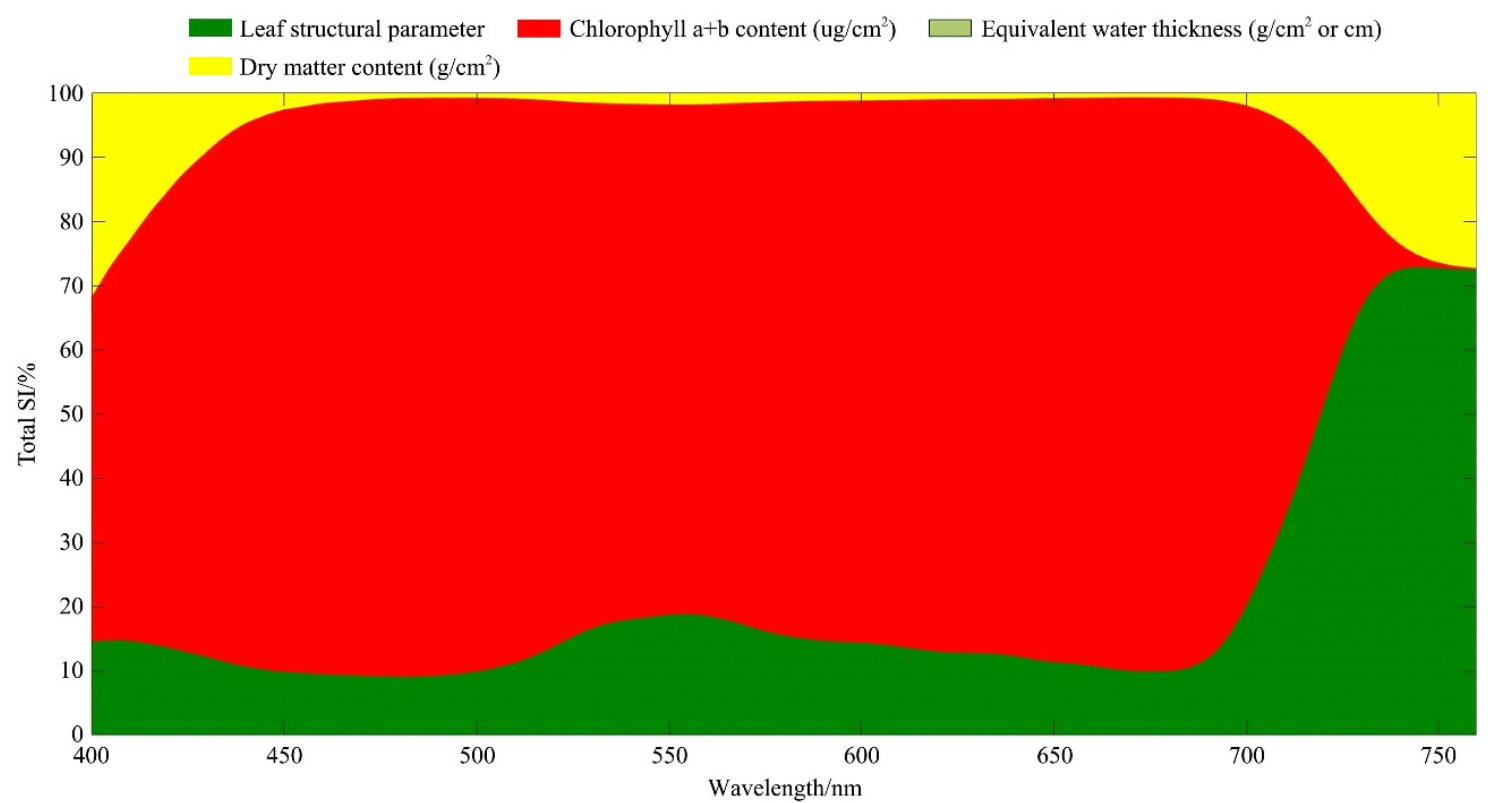

Figure 2 Chlorophyll $\mathrm{a}+\mathrm{b}$ mainly affects the band range

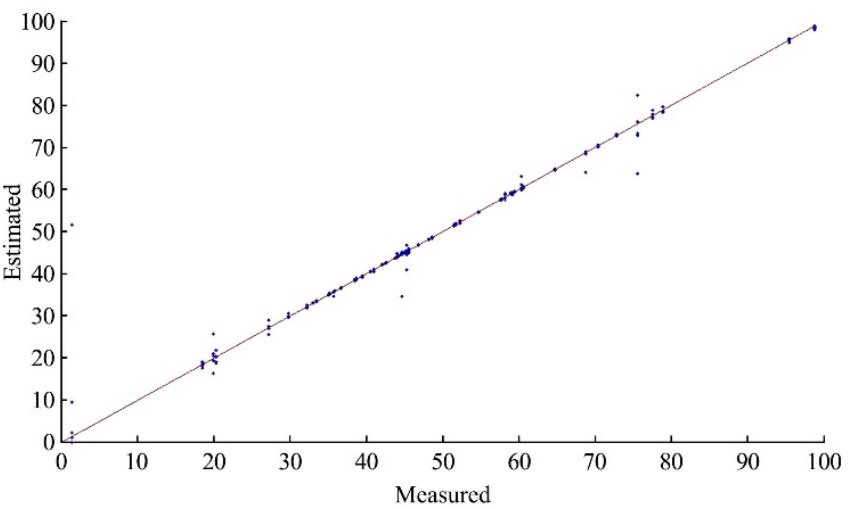

Figure 3 GPR result measured vs established

\subsection{Discussion}

This study analyzed the global sensitivity of the PROSPECT-4 model to find the chlorophyll sensitive bands for establish inversion model. The chlorophyll inversion model was established by GPR method using Lope' 93 leaf biological parameters and hyperspectral datasets. It can be seen from the results that the inversion precision of this study is ideal, which is mainly due to the use of radiative transfer model to link spectral information and crop information, and not the vegetation index as the inversion parameter, which improves the accuracy of the model. The PROSPECT-4 model used in this study did not include other pigment parameters except chlorophyll, which would have some influence on the accuracy of the model. The sensitivity of chlorophyll in crops was $400 \mathrm{~nm}$ to $750 \mathrm{~nm}$. Although chlorophyll was the main parameter affecting the spectral information in this region, other crop parameters also affected the range of this spectral range. The impact of other crop information was not explored in this study.

The data set used in this study is the LOPEX database, which contains crop information and hyperspectral information, and the variety of crops is very generous, is used to explore the crop parameter inversion model of a better database.

The inversion method used in this study is limited by the radiative transfer mechanism model. Only the crop parameters belong to the radiative transfer mechanism model. Therefore, how to establish a radiation transport model that can be used to retrieve crop parameters is the key to improve the accuracy of inversion.

Beyond the here presented remote sensing vegetation products, it would be interesting to apply GPR and RTM to spectral-variable datasets that include more plant properties, such as leaf nitrogen content, biomass.

\section{Conclusions}

In this study, the LOPEX' 93 database was used to combine crop information and hyperspectral information of the modified dataset to establish a hyperspectral information inversion model of crop chlorophyll content through PROSPECT-4 model and GPR method. The main contributions of this research are as follows: (1) The PROSPECT-4 model was analyzed by GSA tool, and the sensitivity band range of crop chlorophyll was at 400-750 nm. (2) the chlorophyll content model was established with great accuracy $\left(R^{2}=0.8638\right)$ that can predict the crop leaf chlorophyll content; (3) The results demonstrated that crop chlorophyll is inversion by PROSPECT model and machine learning algorithm. Therefore, crop chlorophyll content can be estimated by hyperspectral data that may be used for crop growth management.

\section{Acknowledgments}

This work was funded by National Key R\&D Program of Chin a and by Ministry of Science and Technology of the People's Repu blic of China (2016YFD0200600 and 2016YFD0200603).

\section{Supplementary material}

Supplementary data associated with this article can be found, in the online version, at http://teledetection.ipgp.jussieu.fr/ opticleaf/lopex.htm. And http://ipl.uv.es/artmo.

\section{[References]}

[1] Habib, A., Han, Y., Xiong, W., He, F., Zhang, Z., \& Crawford, M. Automated Ortho-Rectification of UAV-Based Hyperspectral Data over an Agricultural Field Using Frame RGB Imagery. Remote Sensing, 2016; 8(10): 1-22.

[2] Fenghua, Y, Tongyu, X, Yingli, C, Guijun, Y, Wen, D, \& Shu, W. Models for estimating the leaf NDVI of japonica rice on a canopy scale by combining canopy NDVI and multisource environmental data in Northeast China. International Journal of Agricultural and Biological 
Engineering, 2016; 9(5): 132-141.

[3] Homolova, L, Malenovský, Z, Clevers, J. G, García-Santos, G., \& Schaepman, M. E. Review of optical-based remote sensing for plant trait mapping. Ecological Complexity, 2013; 15, 1-16.

[4] Khodadadzadeh, M., Li, J., Prasad, S., \& Plaza, A. Fusion of hyperspectral and LiDAR remote sensing data using multiple feature learning. IEEE Journal of Selected Topics in Applied Earth Observations and Remote Sensing, 2015; 8(6): 2971-2983.

[5] Verrelst, J., Rivera, J. P., Tol, C. V. D., Magnani, F., Mohammed, G., \& Moreno, J. Global sensitivity analysis of the scope model: what drives simulated canopy-leaving sun-induced fluorescence. Remote Sensing of Environment, 2015; 166, 8-21.

[6] Yang, H. L, Crawford, M. M. Spectral and spatial proximity-based manifold alignment for multitemporal hyperspectral image classification. IEEE Transactions on Geoscience \& Remote Sensing, 2016; 54(1): 51-64

[7] Barry Haack, Ann Rafter. Regression estimation techniques with remote sensing: a review and case study. Geocarto International, 2009; 25(1): 71-82.

[8] Yu F H, Xu T Y, Cao Y L, Yang G J, Du W, Wang S. Models for estimating the leaf NDVI of japonica rice on a canopy scale by combining canopy NDVI and multisource environmental data in Northeast China. Int J Agric \& Biol Eng,2016; 09(05): 132-142.

[9] Kira, O., Dubowski, Y., \& Linker, R. Reconstruction of passive open-path ftir ambient spectra using meteorological measurements and its application for detection of aerosol cloud drift. Optics Express, 2015; 23(15): 916-29.

[10] Boegh, E., Soegaard, H., Thomsen, A., \& Hansen, S. Multi-scale remote sensing based estimation of leaf area index and nitrogen concentration for photosynthesis modelling. Geoscience and Remote Sensing Symposium, 2003. IGARSS '03. Proceedings. 2003 IEEE International (Vol.4, pp.2847-2849 vol.4). IEEE.

[11] Chappelle, E. W., Kim, M. S., \& Jeiii, M. M. Ratio analysis of reflectance spectra (rars): an algorithm for the remote estimation of the concentrations of chlorophyll a, chlorophyll $\mathrm{b}$, and carotenoids in soybean leaves. Remote Sensing of Environment, 1992; 39(3): 239-247.

[12] Filella, I., Serrano, L., Serra, J., \& Penuelas, J. Evaluating wheat nitrogen status with canopy reflectance indices and discriminant analysis. Crop Science, 1995; 35(5): 1400-1405.

[13] Blackburn, G. A. Quantifying chlorophylls and caroteniods at leaf and canopy scales: an evaluation of some hyperspectral approaches. Remote Sensing of Environment, 1998; 66(3): 273-285.

[14] Baret, F., Buis, S. Estimating Canopy Characteristics from Remote Sensing Observations: Review of Methods and Associated Problems. Advances in Land Remote Sensing. Springer Netherlands. 2008.

[15] Darvishzadeh, R., Skidmore, A., Schlerf, M., \& Atzberger, C. Inversion of a radiative transfer model for estimating vegetation lai and chlorophyll in a heterogeneous grassland. Remote Sensing of Environment, 2008; 112(5): 2592-2604.

[16] Verrelst, J., Rivera, J. P., Leoneko, G., Alonso, L., Moreno, J. Optimizing
LUT-based RTM Inversion for Semiautomatic Mapping of Crop Biophysical Parameters from Sentinel-2 and -3 data: Role of Cost Functions. IEEE Transactions on Geoscience and Remote Sensing, 2014; 52(1): 257-269.

[17] Rivera, J.P., Verrelst, J., Gómez-Dans, J., Muñoz-Marí, J., Moreno, J., Camps-Valls, G. An Emulator Toolbox to Approximate Radiative Transfer Models with Statistical Learning. Remote Sensing, 2015; p. 7 , 9347-9370.

[18] Hosgood, B., Jacquemoud, S., Andreoli, G., Verdebout, J., Pedrini, G., \& Schmuck, G. Leaf optical properties experiment 93 (lopex93). 1994.

[19] Jacquemoud, S., \& Baret, F. Prospect: a model of leaf optical properties spectra. Remote Sensing of Environment, 1990; 34(2): 75-91.

[20] Feret, J. B., François, C., Asner, G. P., Gitelson, A. A., Martin, R. E., \& Bidel, L. P. R., et al. Prospect-4 and 5: advances in the leaf optical properties model separating photosynthetic pigments. Remote Sensing of Environment, 2008; 112(6): 3030-3043.

[21] Jacquemoud, S., Ustin, S. L., Verdebout, J., Schmuck, G., Andreoli, G., \& Hosgood, B. Estimating leaf biochemistry using the prospect leaf optical properties model. Remote Sensing of Environment, 1996; 56(3): 194-202.

[22] Laurent, E. J., Shi, H., Gatziolis, D., Lebouton, J. P., Walters, M. B., \& Liu, J. Using the spatial and spectral precision of satellite imagery to predict wildlife occurrence patterns. Remote Sensing of Environment, 2005; 97(97): 249-262.

[23] Mousivand, A., Menenti, M., Gorte, B., \& Verhoef, W. Global sensitivity analysis of the spectral radiance of a soil-vegetation system. Remote Sensing of Environment, 2014; 145(5): 131-144.

[24] Verrelst, J., Rivera, J.P., Moreno, J. ARTMO's global sensitivity analysis (GSA) toolbox to quantify driving variables of leaf and canopy radiative transfer models. EARSeL eProceedings, Speical Issue 2: 9th EARSeL Imaging Spectroscopy Workshop, 2015; 1-11

[25] Sobol', I. M. On sensitivity estimation for nonlinear mathematical models. Keldysh Applied Mathematics Institute, 1990; 2(1): 112-118.

[26] Verrelst, J., Rivera, J.P., Moreno, J. ARTMO's global sensitivity analysis (GSA) toolbox to quantify driving variables of leaf and canopy radiative transfer models. In: 9th EARSEL SIG Imaging Spectroscopy Workshop, 14-16 April, Luxembourg, 2015.

[27] Williams, Christopher, K. I., Rasmussen, \& Edward, C. Gaussian Processes For Machine Learning. Gaussian processes for machine learning /. MIT Press. 2006

[28] Verrelst, J., Rivera, J. P., Gitelson, A., Delegido, J., Moreno, J., \& Camps-Valls, G. Spectral band selection for vegetation properties retrieval using gaussian processes regression. International Journal of Applied Earth Observation \& Geoinformation, 2016; 52, 554-567.

[29] Rasmussen, J., Sommerlarsen, J., Pedersen, K., Toft, S., Benson, A., \& Bower, R. G., et al. Investigating hot gas in the halos of two massive spirals: observations and cosmological simulations, 2006.

[30] Verrelst, J., Rivera, J.P., Moreno, J. From model simulations towards vegetation properties mapping: automating, optimizing \& simplifying. In: ISSI Workshop on Exploring the Earth's Ecostystems on a Global Scale: Requirements, Capabilities and Directions in Spaceborne Imaging Spectroscopy, 21-25 November, Bern, Switzerland. 2016. 